\title{
BMJ Open Study protocol for the Healthier Wealthier Families (HWF) pilot randomised controlled trial: testing the feasibility of delivering financial counselling to families with young children who are identified as experiencing financial hardship by community-based nurses \\ To cite: Price AMH, Zhu A
} Nguyen HNJ, et al. Study protocol for the Healthier Wealthier Families (HWF) pilot randomised controlled trial: testing the feasibility of delivering financial counselling to families with young children who are identified as experiencing financial hardship by communitybased nurses. BMJ Open 2021;11:e044488. doi:10.1136/ bmjopen-2020-044488

- Prepublication history and additional supplemental material for this paper are available online. To view these files, please visit the journal online (http://dx.doi.org/10.1136/ bmjopen-2020-044488)

Our highly-regarded and wellliked HWF colleague, Prof Margaret Kelaher, sadly passed away in March 2021. We offer our deepest sympathy to her family and friends.

SW and SG are joint senior authors.

Received 06 September 2020 Revised 08 February 2021 Accepted 04 May 2021

Check for updates

(c) Author(s) (or their employer(s)) 2021. Re-use permitted under CC BY-NC. No commercial re-use. See rights and permissions. Published by BMJ.

For numbered affiliations see end of article.

Correspondence to

Dr Anna M H Price;

anna.price@mcri.edu.au

\section{Anna M H Price (D , , ${ }^{1,2,3}$ Anna Zhu, ${ }^{4}$ Huu N J Nguyen, ${ }^{1,2}$ Diana Contreras-Suárez, ${ }^{5}$ Natalie Schreurs, ${ }^{1,2}$ Jade Burley, ${ }^{6,7,8}$ Kenny D Lawson, ${ }^{9}$ Margaret Kelaher (D) , ${ }^{10}$ Raghu Lingam, ${ }^{6,8}$ Rebekah Grace, ${ }^{6,11}$ Shanti Raman, ${ }^{12,13}$ Lynn Kemp, ${ }^{9}$ Susan Woolfenden, ${ }^{6,7,8}$ Sharon Goldfeld (10) 1,2,3}

\section{ABSTRACT}

Introduction Poverty and deprivation can harm children's future health, learning, economic productivity and societal participation. The Australian Healthier Wealthier Families project seeks to reduce the childhood inequities caused by poverty and deprivation by creating a systematic referral pathway between two free, community-based services: universal, well-child nursing services, which provide health and development support to families with children from birth to school entry, and financial counselling. By adapting the successful Scottish 'Healthier Wealthier Children' model, the objectives of this Australian pilot are to test the (1) feasibility of systematising the referral pathway, and (2) short-term impacts on household finances, caregiver health, parenting efficacy and financial service use. Methods and analysis This pilot randomised controlled trial will run in three sites across two Australian states (Victoria and New South Wales), recruiting a total of 180 participants. Nurses identify eligible caregivers with a 6-item, study-designed screening survey for financial hardship. Caregivers who report one or more risk factors and consent are randomised. The intervention is financial counselling. The comparator is usual care plus information from a government money advice website. Feasibility will be evaluated using the number/proportion of caregivers who complete screening, consent and research measures, and access financial counselling. Though powered to assess feasibility, impacts will be measured 6 months post-enrolment with qualitative interviews and questionnaires about caregiver-reported income, loans and costs (adapted from national surveys, for example, the Household, Income and Labour Dynamics in Australia Survey); health (General Health Questionnaire 1, EuroQol five-dimensional questionnaire, Depression, Anxiety, Stress
Strengths and limitations of this study

- 'Healthier Wealthier Families' is the first Australian study to evaluate the feasibility of a systematic referral pathway between universal healthcare and community-based financial counselling for families of young children experiencing financial hardship.

- Delivery in three sites across two Australian states (Victoria and New South Wales) will enable greater scalability to different community and service contexts.

- Prioritisation of families and children who are often missed from services and research, by using flexible data collection strategies and working with interpreters.

- As feasibility is the primary outcome of interest, the study is not powered to demonstrate efficacy and rather will inform a large-scale effectiveness trial.

- The programme logic acknowledges that the impacts of poverty on children are mediated through parental capacity and well-being; however, given the short follow-up period and small sample size, child impacts are measured qualitatively through interviews.

Scale short-form); efficacy (from the Longitudinal Study of Australian Children); and financial service use (studydesigned) compared between arms.

Ethics and dissemination Ethics committees of the Royal Children's Hospital (HREC/57372/RCHM-2019) and South West Sydney Local Health District (2019/ETH13455) have approved the study. Participants and stakeholders 
will receive results through regular communication channels comprising meetings, presentations and publications.

Trial registration number ACTRN12620000154909; prospectively registered. Pre-results.

\section{INTRODUCTION}

\section{Childhood poverty and Australia's social welfare system}

The first years of life are a critical developmental period when children living in or on the verge of poverty are the most vulnerable to its detrimental impacts on future health and well-being, cognitive development, educational attainment, employment and societal participation. ${ }^{12}$ Before the coronavirus disease 2019 (COVID-19) pandemic, one in six Australian children was already living in poverty (defined as living below $50 \%$ of median household income), and one in three was deprived of basic health essentials like adequate fruits and vegetables. $^{3-6}$ Ten per cent of children experienced multiple deprivations or were excluded from activities widely regarded as essential, such as medical and dental treatment, insurance, housing and emergency savings. ${ }^{3-6}$

In April 2020, as a result of the government restrictions enacted to control the spread of COVID-19, the Australian Bureau of Statistics estimated that 2.7 million Australians (almost 20\% of the working population) lost their jobs or hours of work. ${ }^{78}$ Many of these Australians are caregivers to young children who are disproportionately likely to experience multiple material deprivations. ${ }^{9}$ This is especially true for children living in young or one-parent households, with an experience of disability or chronic disease, Aboriginal communities or families who do not speak English. ${ }^{9}$ There is strong international evidence that household financial resources impact child health and development outcomes, although the underlying mechanisms are complex. ${ }^{10}$ Protecting households from income poverty and increasing family finances are key strategies for promoting children's opportunities and life chances. $^{10}$

Australia has a complex social welfare system. Intended as a social safety net, it offers a range of support to families incorporating cash transfers, income support payments, tax concessions, and a variety of community services delivered by federal and state governments. This includes new (temporary) income benefits and services that were rapidly established in response to the COVID-19 pandemic. However, many families face barriers to entitlements, services and supports due to reasons including low financial literacy, lack of awareness of the assistance available, the complex application processes involved and the perceived stigma of receiving assistance. ${ }^{11}{ }^{12}$ These families are also at risk of being targeted by high-cost and harmful debt services, gambling providers and pay-day lenders. ${ }^{13}$ The failure of Australia's policies and services to ameliorate childhood poverty is reflected in its prevalence, which has remained between $14 \%$ and $18 \%$ since the 1990 s. $^{4}$

\section{The potential benefits of financial counselling}

Financial counselling is a promising component of the Australian policy response to increase household income and reduce material deprivation. Services are available to low-income households at no cost through non-profit and community organisations, funded by state and federal governments. ${ }^{1415}$ Financial counsellors can help clients overcome barriers to the services and supports available by helping them understand their legal rights and entitlements, negotiate and manage debt, avoid bankruptcy, prevent litigation and, when necessary, referring to other services such as community legal aid, housing support or personal (eg, mental health), family (eg, violence) and gambling supports. ${ }^{16}{ }^{17}$ Some Australian financial counselling services also provide financial education to build an individual's capacity to understand money and make sound financial decisions $\left(\mathrm{eg},{ }^{18}\right)$.

Clients of financial counselling in Australia report significant short-term benefits, such as debt consolidation and waivers, and uptake of welfare entitlements. ${ }^{17} 19$ A recent evaluation of a community non-profit service reported that over two-thirds of clients had avoided legal action, accessed creditor hardship programmes, and felt their financial situations and literacy had improved. ${ }^{20}$ Importantly, 63\% reported improvement in mental and emotional well-being, and $45 \%$ indicated that their relationships with their families and children had improved. ${ }^{20}$ It is estimated that timely access to this assistance delivers a cost-benefit of $\$ 5$ saved for every $\$ 1$ invested in financial counselling programmes. ${ }^{21}$ International research shows that providing people with information about their eligibility for social welfare, and assistance navigating applications and services, can increase both the uptake of benefits and net income. ${ }^{22-24}$ Importantly, this assistance makes the greatest difference for the people experiencing the highest levels of social adversity. ${ }^{22-24}$

Despite these benefits, financial counselling remains a relatively niche service that is unknown to many Australians. Financial counsellors are different from financial planners, credit service providers or debt consolidators, who can recommend and sell specific financial products and charge fees for services. To legally practise, financial counsellors require accreditation membership with a state-based professional financial counselling association, and completion of a supervised training period and ongoing professional and personal development. Referrals to financial counselling are typically made from social workers in health organisations or by clients who gain awareness of the services and self-refer, and made during periods of acute financial crisis. ${ }^{25}$ The services are not systematically linked with or integrated into other service platforms such as health or education, which means the sector is missing a critical opportunity for delivering much-needed preventative and early intervention financial support. 


\section{Using healthcare to identify and respond to poverty and} deprivation

Healthcare is recognised as a promising avenue for identifying and responding to poverty and deprivation. ${ }^{26-30}$ Likewise, financial and money advice services offer potential for shifting the physical and mental health burdens that commonly occur with, and are exacerbated by, financial hardship. ${ }^{31}$ In Scotland, the National Health Service in Greater Glasgow and Clyde (NHSGGC) has been championing the use of primary healthcare for promoting financial inclusion and reducing the structural inequities that lead to poor physical and mental health for families and children. ${ }^{32}$ In 2010, the NHSGGG established the 'Healthier Wealthier Children' (HWC) programme to create new information and referral pathways between the early years health workforce (midwives and health visitors) and money advice workers. ${ }^{33}$

Initially, the HWC project was delivered in 10 Glasgow communities between November 2010 and March 2012, providing personalised financial counselling to pregnant women and families with young children, including information on welfare entitlements, income maximisation, debt reduction, savings accounts and mentored loans. ${ }^{33}$ In 2012, HWC was evaluated for its economic and social impact. Of 2516 families referred, 1347 accessed the programme, and the resulting monetary gain for women and families totalled $£ 3$ million. The authors reported positive spill-over effects including improved health, housing and quality of life. ${ }^{33}$ Implementation of the service model has been sustained and integrated into the Scottish Government policy to reduce poverty since formal project closure in 2013. As of December 2019, the programme has resulted in a cumulative total of 24470 referrals and £34 699481 total financial gain for women and families. ${ }^{34}$

The HWC model shows the increased income and well-being that a systematic referral pathway between healthcare and social welfare can generate for families and young children. ${ }^{29}$ This is consistent with the broader body of literature showing that welfare advice and income maximisation programmes delivered through healthcare can create financial benefits for people with low income. ${ }^{3035}$ However, no studies have replicated the HWC model using universal healthcare for families with young children, and the model is yet to be rigorously evaluated via randomised controlled trial (RCT) design. Systematic reviews of the published literature highlight both the limited quantitative research investigating the impact of welfare advice on health and social outcomes, ${ }^{35-37}$ as well as the poor quality or lack of data investigating whether health services can be harnessed to change individuals' social or economic outcomes. ${ }^{2638}$

\section{The current study}

To develop this evidence, the current study-'Healthier Wealthier Families' (HWF) — will adapt the Scottish HWC model for the Australian context by linking universal, community-based, well-child nursing services that specifically support families with young children from birth to school entry (known as Child and Family Health $(\mathrm{CFH})$ ) with community-based financial counselling services. Despite the well-established impacts of poverty on children's lifelong health and well-being, no Australian CFH service at the state or territory level systematically asks about or responds to poverty or deprivation. The need for a universal response to financial hardship will only increase as the negative impacts of COVID-19 on family finances and mental health unfold.

The study design is a pilot RCT comparing referral to a financial counselling service (the intervention) with money advice information that is freely available on the independent and government-funded website 'MoneySmart' (the comparator/control). The primary objective is to test the feasibility of systematising the identification and referral pathway between the CFH and financial counselling services for families of young children at risk of or experiencing poverty and deprivation. This primary outcome is assessed with uptake data, and not a between-groups comparison of the RCT. The secondary objectives are to explore the short-term impacts of the intervention on household finances, caregiver health, parenting efficacy and use of financial services, and will be evaluated via a superiority trial design with two parallel groups and a primary endpoint at 6 months (noting the sample size is designed to test feasibility and underpowered to test efficacy), in conjunction with qualitative interviews.

This feasibility RCT is a first step toward a definitive multisite effectiveness trial to examine whether the HWF intervention could reduce the impact of poverty and deprivation for Australian families when delivered at scale. While the pilot was not originally planned with the COVID-19 pandemic in mind, the catastrophic effects of the pandemic on family finances and well-being mean it is increasingly important to refine the feasibility and processes of this potential intervention.

\section{METHODS AND ANALYSIS}

The protocol is written adhering to the Standard Protocol Items: Recommendations for Interventional Trials guidelines (see online supplemental file). The research collaborators use an ongoing process review following quality improvement principles to refine the protocol. Notably, this feasibility trial is adapting to the changing social restrictions resulting from the COVID-19 pandemic, which differ across Australia. Table 1 presents the trial overview.

\section{Study setting}

This feasibility RCT is conducted in three Australian local government areas. These include two rapidly growing Victorian areas, one regional and one metropolitan in the capital city of Melbourne, and one metropolitan area in Sydney, the capital city of New South Wales. Participants are recruited from the free and universally available 
Table 1 Overview of the Healthier Wealthier Families pilot randomised controlled trial (RCT)

Randomisation unit Individual

Sample size

Each of the three sites anticipates enrolling 60 participants (30 per arm). The total target sample size is therefore 180 participants (90 per arm) with an anticipated 135 (75\%) providing 6-month data. As reported by Teare et al, who assessed the sample size requirements of external pilot RCTs, this sample size will be sufficient to estimate the critical parameters of continuous or binary primary outcomes for the definitive RCT (of $n=70 / 120$ for continuous/binary outcomes), including poverty or poor parental mental health. ${ }^{46}$ The sample size was chosen pragmatically and in discussion with the participating sites as being possible relative to the study duration.

Intervention arm Financial counselling

Control arm Usual care plus money advice information

Inclusion criteria

- The parent or caregiver of a young child aged from birth to 5 years old.

- A client of the participating CFH services.

- Living inside the geographical boundaries serviced by the participating financial counselling organisations.

- Reporting at least one risk factor for financial hardship, as screened by the CFH nurse (see box 1).

The research is designed to include families with low literacy and who speak languages other than English. All communication can be conducted by phone or video, and interpreters are engaged to support families as required/requested. Due to the constraints of the pilot funding and resources, participating caregivers in the culturally diverse NSW site need to be fluent in at least one of the four most common languages: English, Arabic, Assyrian and Vietnamese. ${ }^{47}$ The main study documents will also be translated into these languages to increase engagement with potential participants.

$\begin{array}{ll}\text { Exclusion criteria } & \text { Participants meeting any of the following criteria at screening or enrolment are excluded from the pilot } \\ \text { study: } & \text { Do not comprehend the recruitment invitation using phone/video communication and interpreters as required/ } \\ \text { requested (eg, due to substantial cognitive disability). } \\ \text { Already enrolled and assigned a research participant ID. } \\ \text { Participants identified as high risk for high-impact consequences (ie, assets or income at immediate } \\ \text { risk, loss of accommodation or loss of access to essential utilities likely) are not randomised; this } \\ \text { group (termed 'priority 1') is referred directly to financial counselling. For those who consent, research } \\ \text { data are collected to inform the feasibility of the study. } \\ \text { Outcome of } & \text { Definite Go ('green light') defined as: } \\ \text { feasibility trial for } & \geq 50 \% \text { of eligible participants consenting to pilot trial. } \\ \text { progressing to large- } & \geq 60 \% \text { of those in the intervention arm receiving the intervention. } \\ \text { scale trial } & \text { Definite Stop ('red light') defined as: } \\ \text { (Stop-Go criteria) } & <30 \% \text { of eligible participants consenting to pilot trial. } \\ & <30 \% \text { of those in the intervention arm receiving the intervention. }\end{array}$

Primary outcome: feasibility

Number/proportion of:

- Potential participants who agree to complete screening survey (nurse report based on service records).

- Eligible clients who consent to participate (nurse and study records).

- Intervention participants who access, attend and complete the financial counselling intervention (financial counsellor report, participant report).

- Participants who complete the 6-month follow-up data collection questionnaire (collected by phone or in person) (study completion records).

Secondary

outcomes: impact
Measured at 3 and 6 months post-enrolment via participant report:

1. Household income, sources of income and types of loans, measured using questions adapted from the Household, Income and Labour Dynamics in Australia (HILDA) ${ }^{39}$ Survey and Longitudinal Study of Australian Children (LSAC). ${ }^{40}$

2. Meeting household costs, using questions adapted from the HILDA ${ }^{39}$ Survey, Australian Research Alliance for Children and Youth Nest Survey, ${ }^{3}$ and Community Understanding of Poverty and Social Exclusion Survey. ${ }^{41}$

3. Caregiver general health, measured using the General Health Questionnaire $1^{48} 49$ and the EuroQol fivedimensional questionnaire. ${ }^{4350}$

4. Caregiver mental health, measured using Depression, Anxiety, Stress Scale short-form. ${ }^{51}$

5. Parenting efficacy, using questions from the LSAC. ${ }^{40}$

6. Use of financial services, measured with study-designed questions.

And at 6 months post-enrolment via interview:

7. Nurse, financial counsellor and caregiver views on the feasibility of the research processes, and impacts on caregivers and children. 


\begin{tabular}{ll}
\hline Table 1 Continued & \\
\hline $\begin{array}{l}\text { Randomisation } \\
\text { procedure }\end{array}$ & $\begin{array}{l}\text { A statistician prepares the randomisation schedule using block randomisation. Participants are } \\
\text { randomly assigned to either control or intervention arm with a 1:1 allocation following a computer- } \\
\text { generated randomisation schedule stratified by site, using permuted blocks. }\end{array}$ \\
\hline Blinding (masking) & The researchers assessing the outcomes and analysing the data are blind to randomisation status. \\
\hline
\end{tabular}

CFH, Child and Family Health; NSW, New South Wales.

CFH nursing services (also known as the Maternal and Child Health service in some states), which are governed at the state and local levels. Nurses in both states work with interpreters by phone or in person for appointments with families who speak languages other than English. Figure 1 presents the study timeline.

\section{Interventions}

The purpose of financial counselling intervention is to help each family maximise their income, reduce debt and help with budgeting. Thus, the intervention is tailored to a participant's personal and financial needs. Before the COVID-19 pandemic, financial counsellors typically worked with clients over the phone or at the counsellors' offices. In some instances of access issues, they visited a client in their home. During the COVID-19 pandemic, the participating financial counsellors predominately deliver their services via phone. For non-English-speaking clients, it is usual practice for the financial counsellors to deliver the service via a multilingual counsellor, or in conjunction with an independent telephone interpreter.

Following allocation to the intervention, a referral with the participant's contact information is emailed to the financial counsellor intake address, and a financial counsellor is assigned. The participant is contacted to arrange the first intervention appointment, which includes confirming contact and demographic details; ascertaining capacity to make financial decisions; discussing and prioritising financial needs; and planning for working together. Clients are asked to gather and send paperwork to financial counsellors or external agencies and are assisted by

\begin{tabular}{|l|c|c|c|c|c|c|}
\hline TRIAL PERIOD & Screening & Enrolment & Allocation & \multicolumn{2}{|c|}{$\begin{array}{c}\text { Post- } \\
\text { allocation }\end{array}$} & $\begin{array}{c}\text { Close- } \\
\text { out }\end{array}$ \\
\hline IIME POINT & $-\boldsymbol{t}_{2}$ & $-\boldsymbol{t}_{1}$ & $\mathbf{0}$ & $\boldsymbol{t}_{1}$ & $\boldsymbol{t}_{2}$ & $\boldsymbol{t}_{3}$ \\
\hline ENROLMENT & & & & & & \\
\hline Eligibility screen & $\mathbf{X}$ & & & & & \\
\hline Informed consent & & $\mathbf{X}$ & & & & \\
\hline Enrolment questionnaire & & $\mathbf{X}$ & & & & \\
\hline Intervention allocation & & & $\mathbf{X}$ & & & \\
\hline INTERVENTIONS & & & & & & \\
\hline $\begin{array}{l}\text { Intervention: Financial } \\
\text { counselling }\end{array}$ & & & & $\mathbf{X}$ & $\mathbf{X}$ & $\mathbf{X}$ \\
\hline $\begin{array}{l}\text { Usual care/Control: } \\
\text { Information }\end{array}$ & & & & $\mathbf{X}$ & & \\
\hline $\begin{array}{l}\text { FOLLOW-UP } \\
\text { QUESTIONNAIRES }\end{array}$ & & & & & & \\
\hline $\begin{array}{l}\text { Demographics } \\
\text { Secondary outcomes }\end{array}$ & & & & & $\mathbf{X}$ & $\mathbf{X}$ \\
\hline Intervention fidelity & & & & & $\mathbf{X}$ & $\mathbf{X}$ \\
\hline $\begin{array}{l}\text { Semi-structured } \\
\text { interviews }\end{array}$ & & & & & & $\mathbf{X}$ \\
\hline
\end{tabular}

Figure 1 Participant timeline. $t_{1}$ : within 2 weeks postenrolment; $\mathrm{t}_{2}$ : 3 months post-enrolment; $\mathrm{t}_{3}$ : 6 months postenrolment. the financial counsellor to complete forms in a timely and accurate manner. Financial counsellors often contact and negotiate with creditors on a client's behalf. Each participant has as many visits with the financial counsellor as they need; these are scheduled together and confirmed by phone calls and text messages.

The comparator is usual care plus money advice that is freely available on the federally funded Australian Securities and Investments Commission's MoneySmart website (the control). MoneySmart provides objective, plain-language advice developed by financial counsellors on a comprehensive range of debt, budgetary management and financial literacy topics, and selected resources are available in multiple languages. MoneySmart outlines methods and contact details for further financial, legal and crisis support; this includes the National Debt Helpline, a free hotline which links callers to a local financial counselling service. MoneySmart is commonly used by Australian financial counsellors to advise clients. While the comparator offers valuable but passive information via a website, it is distinct from the intervention, which operationalises this information for eligible families by tailoring support to their immediate financial needs and challenges.

\section{Recruitment and consent}

Potential participants are individuals who have an appointment with a participating $\mathrm{CFH}$ nurse and fulfil the eligibility criteria (figure 2). The nurse managers (study partners) select the nurses and centres who conduct screening. Participating nurses complete a short screening survey with clients at their appointments. Box 1 describes the screening items which were co-designed by the HWF collaborating partners. Items were drawn from national and local datasets describing the characteristics most likely to identify families with young children who are at risk of or experiencing poverty and deprivation. ${ }^{39-41}$

If the screening survey shows that a client is eligible for the study, the nurse mentions this and asks if the client would like more information. If a client agrees, nurses note this, check and record the client's contact information on the survey form, and explain that they will pass the client's contact details onto the research team who will contact them. If a client says no, nurses record a reason where one is provided and return the screening data to the research team without recording the client's identifying information. Surveys are collected until the necessary sample size for each site is reached or the recruitment period ends. 


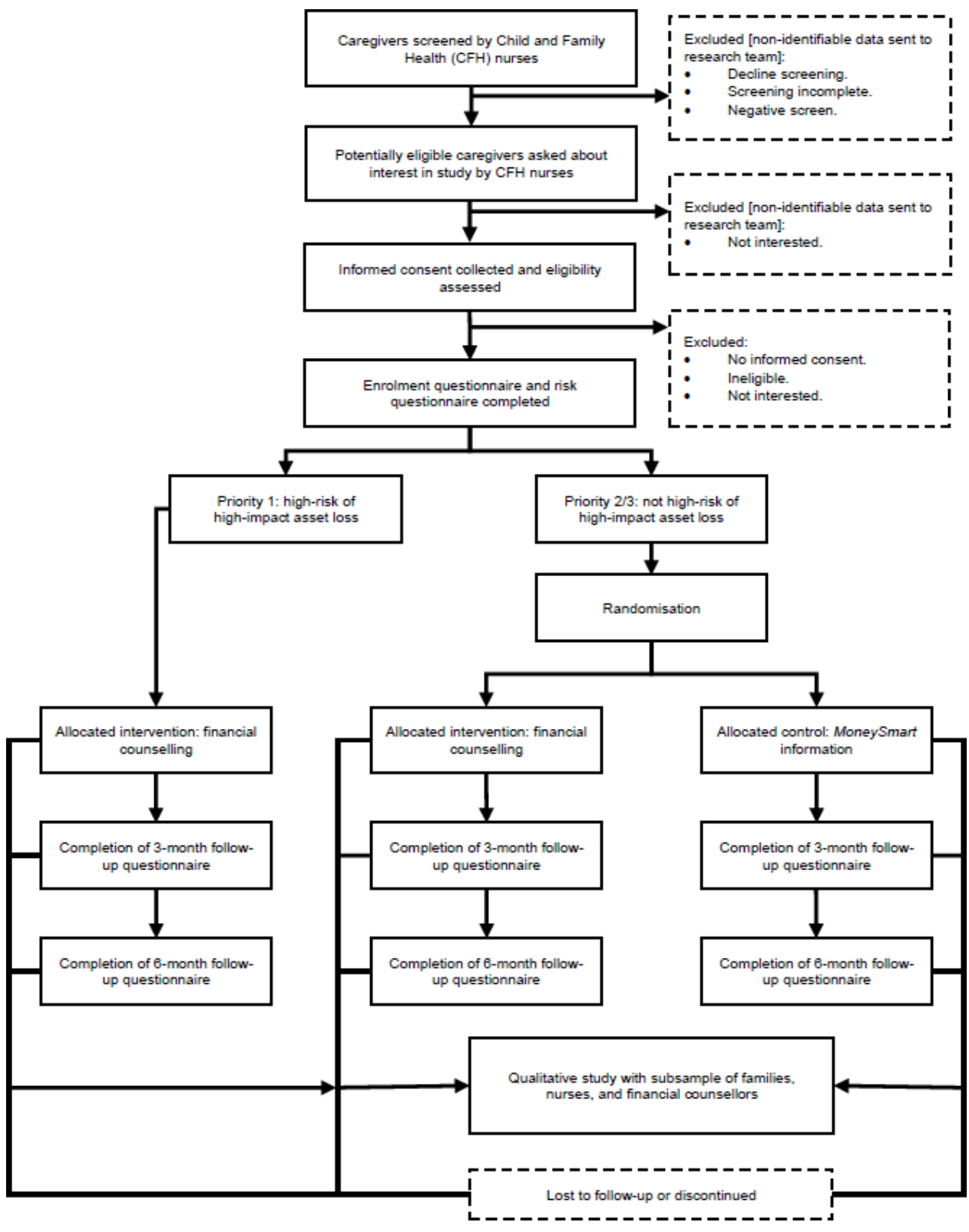

Figure 2 Participant journey.

Eligible clients are welcomed to the study by the research team via phone call or email within a week of the team receiving the screening survey. Over the phone, a researcher introduces the study and answers any questions that potential participants have about the study.

\section{Box 1 Financial hardship risk factors for eligibility}

Item 1. No one in household currently has a paid job.

Item 2 . The family does not have at least $\$ 500$ in savings for an emergency today.

In the last year, because of money pressure, the respondent missed or put off:

Item 3. Mortgage or rent repayments.

Item 4. Paying electricity, gas, water bills.

Item 5. Buying prescription medicines.

Item 6. Paying home or car insurance.
Interpreters are engaged for phone calls and visits as indicated on the screening survey or identified during phone calls. The study welcome email includes the Participant Information and Consent Form (PICF) and enrolment questionnaire. Participants can provide informed consent by: (1) clicking the relevant consent link on the electronic version and following the database prompts; (2) signing the electronic version and returning to the study email or by post, (3) verbally on the phone or by video link, or when face-to-face visits are possible and safe, (4) signing the hardcopy in person with the researcher.

\section{Allocation, concealment and implementation}

Participants are randomised using a study-designed, online, central randomisation service that stratifies by site. Randomisation occurs only after a participant provides informed consent, the eligibility of the participant is 
confirmed and enrolment is complete. The randomisation schedule is securely stored by the site-specific randomisation programme and concealed by the statistician engaged by the research study for this purpose.

\section{Blinding}

The researchers responsible for data collection and data analysis are blinded to treatment allocation. Participants are asked not to disclose their randomisation status during data collection questionnaires. Where a researcher is unblinded, this is recorded by the manager for consideration in the impact analyses.

The research partners who are not blinded include: the research managers and participants who are aware of randomisation to enable allocation and intervention delivery. The financial counsellors are informed who the intervention participants are but do not know who the control participants are, unless control participants seek the services of a financial counsellor themselves (as can happen in usual care), and disclose participation in the research study. The nurses are not informed of allocation but may become aware if participants disclose it, or the research team seeks a nurse's advice to respond to a support issue involving the participant.

\section{Quantitative data collection methods}

The processes are designed to carefully and respectfully consider the needs of participants who may have complex lives or low English proficiency, for whom more research effort is necessary to contact, recruit and retain. ${ }^{42}$ The pilot therefore includes a range of options for consent and data collection, including online, by phone, by video link or by post, and with support from interpreters. Where it is safe, questionnaires can also be completed with participants in their homes. Participants will be invited to complete follow-up questionnaires at 3 and 6 months post-enrolment to assess the primary and secondary outcomes described in table 1 . At the same time points, financial counsellors will be invited to complete a fidelity questionnaire for each intervention client to assess the goals initially set; frequency of participant contact and compliance; specific activities and advice delivered; and known changes in participant financial status.

\section{Qualitative data collection methods}

At 6 months post-enrolment, following collection of the 6-month follow-up questionnaire, a stratified subsample of caregivers (8-10 across both trial arms in each site) representing partnered and sole parents, cultural diversity and those with lower or higher numbers of risk factors for financial hardship, CFH nurses (one to four per site) and financial counsellors (one to four per site), who consent to interview, will be purposefully chosen to complete a 30-minute semistructured interview by phone/video. Families and financial counsellors will be asked for their perceptions of financial supports (including the intervention) used during the study period, and the impacts on families and children. Nurses will be asked for their perceptions of screening and recruitment processes for identifying and engaging families who could benefit from financial counselling.

\section{Data management}

All participants are assigned a unique numerical identifier for use throughout the study. Secure electronic databases record all participant data. Hardcopy materials that cannot be transferred to electronic documents are stored in locked cabinets. Only project staff directly involved in the analysis of the data have access to data. All project data are stored for the required period, that is, indefinitely if the participant consents to providing their data for data pooling or, otherwise, for 15 years after the completion of the study. After that time, hardcopy materials are destroyed by shredding, and any password-protected electronic archives are permanently deleted.

\section{Statistical analysis methods}

Baseline characteristics of participating families will be described for each trial arm using the mean, median and IQR for continuous data and proportions for categorical data. Primary outcomes are the feasibility measures described in table 1 and will be assessed descriptively. Secondary outcomes are the impact measures in table 1 and will be described by treatment arm. As this is a feasibility pilot, the sample size is not calculated to have sufficient power to detect between-groups differences in the secondary outcomes. Rather, we will explore impact and inform the sample size for a large-scale RCT by comparing groups using the t-test and non-parametric tests for continuous data and the $\mathrm{X}^{2}$ test for categorical data. Australian valuation weights for the EuroQol five-dimensional questionnaire will be applied to generate health utility scores, ${ }^{43}$ and the incremental difference between trial arms estimated. Linear and logistic regression modelling will be conducted to test group differences adjusting for basic baseline characteristics identified a priori (where the model allows), which may differ between sites and group allocation; for example, participant gender, age, mental health and sociodemographic characteristics. All regression analyses will be adjusted for study site in line with the stratification of the study randomisation. The only subgroup analysis planned is to describe the participants identified as priority 1 (high-risk) who are not included as RCT participants but do continue as research participants. Their data will be described using the above methods.

\section{Qualitative analysis methods}

Semistructured interviews will be audio-recorded and transcribed using Rev software or Zoom transcription function. Transcriptions will be reviewed by the researcher for accuracy. Data will be entered into NVivo for management, will be thematically coded with constant comparison between transcripts and analysed using Braun $e t a l$ 's 6 -phase reflexive approach. ${ }^{44}$ These data will be used to develop and refine the HWF programme logic 
and protocol, with the broader goals of maximising the programme feasibility and effectiveness for the large-scale trial.

\section{Patient and public involvement}

Before screening or intervention delivery commences, the nurses and financial counsellors ask non-participant clients for feedback on the screening tool and trial goals, which is integrated into the study processes. As part of the trial's ongoing process review, the researchers, nurses and financial counsellors seek consumer feedback on the trial processes to understand and refine the feasibility of the study processes.

\section{Data monitoring and auditing}

The HWF Advisory Groups incorporate key stakeholders and experts to direct and advise on the technical design, implementation and interpretation of the feasibility trial. No data monitoring committee is needed due to the minimal risks of the research. No audits are planned, although the governing ethics committees may audit this research as part of their own processes. No interim analyses or stopping rules will be applied, although the pilot may be terminated early if recruitment or intervention delivery is not feasible due to social interventions resulting from COVID-19 or another epidemic or catastrophe.

Table 1 describes the Stop-Go criteria to progress from the pilot to large-scale RCT. These criteria are informed by nurses' and financial counsellors' advice on anticipated recruitment and intervention delivery, the challenges posed to service delivery by the COVID-19 pandemic (such as the rapid transitioning from face-toface to phone appointments, and the barriers to access that these changes can create for eligible families), and the team's experience conducting longitudinal and intervention research with families experiencing adversity. ${ }^{42}$ Areas that are amenable to change before a large-scale trial will be investigated as highlighted by Charlesworth $e t$ $a l,{ }^{45}$ through the qualitative study.

\section{Managing potential harms}

Adverse events are recorded from the time the participant completes informed consent until 30 days after the last study visit. It is anticipated that adverse events will most likely relate to psychosocial, substance abuse, home safety and child protection issues. The HWF Safety and Communication Protocol provides researchers with a structured set of responses to participant-disclosed risks including support needs, safety related and emergencies. Support and debriefing for research staff are provided by the investigators and employee assistance programmes. Where an incident that requires a child protection notification occurs during a nurse or financial counsellor visit, the nurses and financial counsellors will respond in accordance with their local mandatory reporter guidelines.

\section{ETHICS AND DISSEMINATION}

The study is approved by the Human Research Ethics Committees of the Royal Children's Hospital (HREC/57372/RCHM-2019), and South West Sydney Local Health District (2019/ETH13455). A condition of approval is that any proposed amendments to the project, including changes to the protocol, PICF and participant materials are submitted to the governing HRECs for approval before use. Protocol amendments are reflected in trial registry updates. The managerial research staff make safety and progress reports to the HRECs at least annually and within 3 months of study termination or completion at each site.

\section{Confidentiality}

Participant confidentiality is strictly held in trust by the investigators, research staff, and the sponsoring institutions and their agents, and is extended to cover clinical information relating to participants. No information concerning the study or data is released to any unauthorised party without prior written approval of the sponsoring institution. Investigators and students have access to the dataset via permissions maintained by the data managers.

\section{Access to data}

All investigators will be able to access cleaned study data for analysis. Data will be housed on the sponsors' secure networks or the file transfer protocol site created for the study, and all datasets will be password protected. To ensure confidentiality, dispersed data files will not include identifying participant information.

\section{Ancillary and post-trial care}

None is planned as the research is low risk; the intervention is an existing service that is already freely available and designed for individuals that include the families targeted by this research; identification of eligible families is conducted by a nursing service that has as part of its core business the identification of issues and referral to relevant agencies; the comparator is usual care practice plus some additional, low-risk money advice information; and the project team is experienced in considering and minimising the risks of this type of intervention research for the participant group.

\section{Dissemination}

The investigators and sponsors will communicate trial results to stakeholders, participants, healthcare professionals, financial counsellors, the public and other relevant groups via meetings, presentations and publications. Data and document sharing will be decided on a caseby-case basis at the discretion of the research team and consistent with ethical approval.

\section{Author affiliations}

${ }^{1}$ Policy and Equity Group, Murdoch Children's Research Institute, Parkville, Victoria, Australia

${ }^{2}$ Centre for Community Child Health, The Royal Children's Hospital Melbourne,

Parkville, Victoria, Australia

${ }^{3}$ Department of Paediatrics, The University of Melbourne, Parkville, Victoria, Australia 
${ }^{4}$ School of Economics, Marketing and Finance, RMIT University, Melbourne, Victoria, Australia

${ }^{5}$ Melbourne Institute: Applied Economic \& Social Research, The University of Melbourne, Melbourne, Victoria, Australia

${ }^{6}$ BestSTART-South West, Ingham Institute, Liverpool, New South Wales, Australia ${ }^{7}$ Sydney Children's Hospital Network, Sydney, New South Wales, Australia

${ }^{8}$ Population Child Health Research Group, School of Women and Children's Health, University of New South Wales, Randwick, New South Wales, Australia

${ }^{9}$ Translational Health Research Institute, Western Sydney University, Penrith South, New South Wales, Australia

${ }^{10} \mathrm{Centre}$ for Health Policy, The University of Melbourne, Melbourne, Victoria,

Australia

${ }^{11}$ Centre for the Transformation of early Education and Child Health, Western Sydney University, Campbelltown, New South Wales, Australia

${ }^{12}$ Community Paediatrics, South Western Sydney Local Health District, Liverpool, New South Wales, Australia

${ }^{13}$ School of Medicine, University of New South Wales, Randwick, New South Wales, Australia

\section{Twitter Anna M H Price @amhprice}

Acknowledgements We thank all families, children, nurses and financial counsellors who are working together on Healthier Wealthier Families (HWF), and members of the HWF Advisory Groups for their oversight and guidance. The HWF collaboration is led by the Centre for Community Child Health and BestSTART-SW, in partnership with Victorian Maternal and Child Health services, NSW Child and Family Health services, Uniting Vic.Tas, Upper Murray Family Care, Wesley Mission, the Melbourne Institute: Applied Economic \& Social Research, the University of Melbourne, RMIT University, Western Sydney University, and the University of New South Wales.

Contributors AMHP contributed to the conception and design of the work; wrote the first draft of the paper, revised it critically and approved the final version; and will be involved in data analysis, interpretation and dissemination. AZ contributed to the conception and design of the work; revised the paper critically and approved the final version; and will be involved in data analysis, interpretation and dissemination. JN contributed to the design of the work; revised the paper critically and approved the final version. DC-S contributed to the design of the work; revised the paper critically and approved the final version; and will be involved in data analysis, interpretation and dissemination. NS contributed to the design of the work; revised the paper critically and approved the final version; and will be involved in data collection; interpretation and dissemination. JB contributed to the design of the work; revised the paper critically and approved the final version; and will be involved in data collection; interpretation, reporting and dissemination. KDL contributed to the design of the work; revised the paper critically and approved the final version; and will be involved in data analysis, interpretation and dissemination. MK contributed to the design of the work; revised the paper critically and approved the final version; and will be involved in data interpretation and dissemination. RL contributed to the design of the work; revised the paper critically and approved the final version; and will be involved in data interpretation and dissemination. RG contributed to the design of the work; revised the paper critically and approved the final version; and will be involved in data interpretation and dissemination. SR contributed to the design of the work; revised the paper critically and approved the final version; and will be involved in data interpretation and dissemination. LK contributed to the design of the work; revised the paper critically and approved the final version; and will be involved in data interpretation and dissemination. SW contributed to the design of the work; revised the paper critically and approved the final version; and will be involved in data interpretation and dissemination. SG contributed to the conception and design of the work; revised the paper critically and approved the final version; and will be involved in data interpretation and dissemination. All authors agree to be accountable for all aspects of the research.

Funding The Victorian site is supported by the Helen Macpherson Smith Trust Impact Grant \#9523 and the Murdoch Children's Research Institute (MCRI). MCRI administered the research grant for the Victorian pilot site and provided infrastructural support (as study sponsor) to its staff but played no role in the conduct or analysis of the trial. Research at the MCRI is supported by the Victorian Government's Operational Infrastructure Support Programme. The New South Wales (NSW) site is supported by The Corella Fund, a Health@Business and University of NSW (UNSW) Medicine Collaboration Seed Funds Grant, the Population Child Health Group at the UNSW, and the BestSTART-SW at the Ingham Institute. The Population Child Health Group at the UNSW and the BestSTART-SW at the Ingham provided the research associate (author JB) and infrastructural support (as study sponsor) to its staff. Author SW was supported by a National Health and Medical Research Council (NHMRC) Career Development Fellowship (\#1158954). Author SG was supported by an NHMRC Practitioner Fellowship (\#1155290).

Competing interests None declared.

Patient consent for publication Not required.

Provenance and peer review Not commissioned; externally peer reviewed.

Supplemental material This content has been supplied by the author(s). It has not been vetted by BMJ Publishing Group Limited (BMJ) and may not have been peer-reviewed. Any opinions or recommendations discussed are solely those of the author(s) and are not endorsed by BMJ. BMJ disclaims all liability and responsibility arising from any reliance placed on the content. Where the content includes any translated material, BMJ does not warrant the accuracy and reliability of the translations (including but not limited to local regulations, clinical guidelines, terminology, drug names and drug dosages), and is not responsible for any error and/or omissions arising from translation and adaptation or otherwise.

Open access This is an open access article distributed in accordance with the Creative Commons Attribution Non Commercial (CC BY-NC 4.0) license, which permits others to distribute, remix, adapt, build upon this work non-commercially, and license their derivative works on different terms, provided the original work is properly cited, appropriate credit is given, any changes made indicated, and the use is non-commercial. See: http://creativecommons.org/licenses/by-nc/4.0/.

\section{ORCID iDs}

Anna M H Price http://orcid.org/0000-0002-8117-8059

Margaret Kelaher http://orcid.org/0000-0002-9899-858X

Sharon Goldfeld http://orcid.org/0000-0001-6520-7094

\section{REFERENCES}

1 Duncan GJ, Ziol-Guest KM, Kalil A. Early-childhood poverty and adult attainment, behavior, and health. Child Dev 2010;81:306-25.

2 Goldfeld S, O'Connor M, Chong S, et al. The impact of multidimensional disadvantage over childhood on developmental outcomes in Australia. Int J Epidemiol 2018;47:1485-96.

3 Sollis K. Measuring child deprivation and opportunity in Australia: applying the nest framework to develop a measure of deprivation and opporutnity for children using the longitudinal study of Australian children. Canberra, 2019.

4 Australian Council of Social Services (ACOSS). Poverty in Australia. Sydney: ACOSS, 2018.

5 Australian Early Development Census. National report 2018: a snapshot of early childhood development in Australia. Canberra: Commonwealth of Australia, 2018.

6 Productivity Commission. Rising inequality? A stocktake of the evidence. Canberra: Productivity Commission Research Paper, 2018.

7 Australian Bureau of Statistics. People who lost a job or were stood down: flows analysis, 2020.

8 Callander EJ, Schofield DJ. Multidimensional poverty and health status as a predictor of chronic income poverty. Health Econ 2015;24:1638-43.

9 Wilkins R. The household, income and labour dynamics in Australia survey: selected findings from waves 1 to 14: the 11th annual statistical report of the HILDA survey. Melbourne: Melbourne Institute, The University of Melbourne, 2016.

10 Cooper K, Stewart K. Does money affect children's outcomes? An update, CASEpaper 203. London: Centre for Analysis of Social Exclusion (CASE), The London School of Economics and Political Science, 2017.

11 Baker D. Missing out: unclaimed government assistance and concession benefits, policy brief No. 14. Canberra (AU: The Australia Institute, 2010

12 Australian and New Zealand Banking Group (ANZ), Social Research Centre. ANZ survey of adult financial literacy in Australia: full report of the results from the 2014 ANZ survey. Sydney: ANZ, 2015 May.

13 Stop the Debt Trap Alliance. The debt trap: how payday lending is costing Australians, 2019.

14 Australian Government Department of Social Services. Financial wellbeing and capability [Website]. Canberra: Australian Government, 2019. Available: https://www.dss.gov.au/communities-andvulnerable-people/programmes-services/financial-wellbeing-andcapability [Accessed 12 Aug 2019].

15 Consumer Affairs Victoria. Financial counselling program 2017-21 [Website]. Melbourne: State of Victoria, 2017. Available: https://www. 
consumer.vic.gov.au/clubs-and-fundraising/funded-services-andgrants/financial-counselling-program [Accessed 12 Aug 2019].

16 Financial Counselling Australia. About financial counselling, 2019 Available: https://www.financialcounsellingaustralia.org.au/aboutfinancial-counselling/

17 Brackertz N. The impact of financial counselling on alleviating financial stress in low income households: a national Australian empirical study. Soc Policy Soc 2014;13:389-407.

18 Zanoni L, Warburton W, Russell R. Evaluating the Wesley Mission "In charge of my money" financial literacy program for vulnerable populations. Sydney: Wesley Mission, 2016.

19 Schetzer L. Drowning in debt: the experiences of people who seek assistance from financial counsellors. Department of Justice, 2007.

20 Brackertz N. I'd Wish l'd Known Sooner! The impact of financial counselling on debt resolution and personal wellbeing. The Salvation Army, 2012.

21 Mahmoudi P, Hordacre A-L, Spoehr J. Paying it forward: cost benefit analysis of the Wyatt trust funded financial counselling services. Adelaide: The Australian Workplace Innovation and Social Research Centre (WISeR), The University of Adelaide, 2014.

22 Deshpande M, Li Y. Who is screened out? Application costs and the targeting of disability programs. Am Econ J Econ Policy 2019;11:213-48.

23 Finkelstein A, Notowidigdo MJ. Take-up and targeting: experimental evidence from SNAP*. Q J Econ 2019;134:1505-56.

24 Armour $\mathrm{P}$. The role of information in disability insurance application: an analysis of the social security statement phase-in. Am Econ J Econ Policy 2018;10:1-41.

25 Price A, Nguyen H. Personal correspondance with R. roles, healthier Wealthier families meeting, 2019.

26 Bibbins-Domingo K. Integrating social care into the delivery of health care. JAMA 2019;322:1763-4

27 Garg A, Toy S, Tripodis Y, et al. Addressing social determinants of health at well child care visits: a cluster RCT. Pediatrics 2015;135:e296-304

28 Rossin-Slater M. WIC in your neighborhood: new evidence on the impacts of geographic access to clinics. J Public Econ 2013;102:51-69.

29 Naven L. Review of healthier, Wealthier children (HWC) in NHS greater Glasgow and Clyde. Glasgow, 2018.

30 Andermann A, CLEAR Collaboration. Taking action on the social determinants of health in clinical practice: a framework for health professionals. CMAJ 2016;188:E474-83.

31 Jones AM, Wildman J. Health, income and relative deprivation: evidence from the BHPS. J Health Econ 2008;27:308-24.

32 Dobbie L, Gillespie M. The health benefits of financial inclusion: a literature review. In: Report for NHS greater Glasgow and Clyde, Scottish poverty information unit. Glasgow Caledonian University, 2010.

33 Naven L, Withington R, Egan J. Maximising opportunities: final evaluation report of the healthier, Wealthier children (HWC) project. report. Glasgow: Glasgow Centre for Population Health, 2012.

34 Price A. Personal correspondance with L Naven, email subject "Question about HWC number of referrals", 2020.

35 Adams J, White M, Moffatt S, et al. A systematic review of the health, social and financial impacts of welfare rights advice delivered in healthcare settings. BMC Public Health 2006;6:81.
36 Allmark P, Baxter S, Goyder E, et al. Assessing the health benefits of advice services: using research evidence and logic model methods to explore complex pathways. Health Soc Care Community 2013;21:59-68.

37 Sherr L, Rodgers A, Varrall R, et al. Examining ways in which contact opportunities associated with transfers might help identify vulnerable households and link them with social welfare services: a systematic review of the literature. Vulnerable Child Youth Stud 2009:4:10-40.

38 Gottlieb LM, Wing H, Adler NE. A systematic review of interventions on patients' social and economic needs. Am J Prev Med 2017;53:719-29.

39 Melbourne Institute. Applied economics \& social research. The household, income and labour dynamics In Australia survey: selected findings from Waves 1 to 17 . Melbourne, Australia: The University of Melbourne, Melbourne Institute, 2019.

40 Australian Institute of Family Studies. Longitudinal study of Australian children data user guide - December 2018. Melbourne: Australian Institute of family studies, 2018. Available: https:// growingupinaustralia.gov.au/data-and-documentation/data-userguide

41 Saunders $\mathrm{P}$, Zhu A. Comparing disadvantage and well-being in Australian families [online]. Aust J Labour Econ 2009;12:21-39.

42 Price $\mathrm{A}$, Bryson $\mathrm{H}$, Smith $\mathrm{A}$, et al. Processes for engaging and retaining women who are experiencing adversity in longitudinal health services research. BMC Health Serv Res 2019;19:833.

43 Viney R, Norman R, King MT, et al. Time trade-off derived EQ-5D weights for Australia. Value Health 2011;14:928-36.

44 Braun V, Clarke V, Hayfield N. Thematic analysis. In: Liamputtong $\mathrm{P}$, ed. Handbook of research methods in health social sciences. Singapore: Springer Singapore, 2019: 843-60.

45 Charlesworth G, Burnell K, Hoe J, et al. Acceptance checklist for clinical effectiveness pilot trials: a systematic approach. BMC Med Res Methodol 2013;13:78.

46 Teare MD, Dimairo M, Shephard N, et al. Sample size requirements to estimate key design parameters from external pilot randomised controlled trials: a simulation study. Trials 2014;15:264.

47 New South Wales Department of Health. South West Sydney: our health - an in-depth study of the health of the population now and into the future, 2018. Available: https://www.swslhd.health.nsw.gov. au/publications.html

48 Raat $\mathrm{H}$, Botterweck AM, Landgraf JM, et al. Reliability and validity of the short form of the child health questionnaire for parents (CHQPF28) in large random school based and general population samples. $J$ Epidemiol Community Health 2005;59:75-82.

49 Waters E, Salmon L, Wake M, et al. The child health questionnaire in Australia: reliability, validity and population means. Aust N Z J Public Health 2000;24:207-10.

50 EuroQol Group. EuroQol--a new facility for the measurement of health-related quality of life. Health Policy 1990;16:199-208.

51 Henry JD, Crawford JR. The short-form version of the depression anxiety stress scales (DASS-21): construct validity and normative data in a large non-clinical sample. Br J Clin Psychol 2005;44:227-39. 